\title{
Investigation of the electron emission from pores in a diamond porous membrane
}

\author{
V. P. Mammana a) \\ Physics Institute, University of Sao Paulo-Sao Paulo, Brazil \\ S. Anders ${ }^{b)}$ and O. R. Monteiro \\ Lawrence Berkeley Laboratory, Berkeley, California \\ M. C. Salvadoric) \\ Physics Institute, University of Sao Paulo-Sao Paulo, Brazil
}

(Received 12 December 1999; accepted 6 June 2000)

\begin{abstract}
It was previously proposed that porous diamond membranes can be used as field emission arrays. As main features of such a device, we identified a field enhancement factor equivalent or superior to that of a metal edge emitter, allied to a higher vacuum conductance. Emitting membrane prototypes were built and tested. These showed promising emission performance. In this work we continue the membrane pore characterization from a geometric point of view. We also present photoemission electron microscopy images from the emitting area around a pore. These images show that indeed there is enhanced electron emission occurring in that region. Current versus time and current versus field graphs complete the emission characterization. (c) 2000 American Vacuum Society.

[S0734-211X(00)00105-0]
\end{abstract}

\section{INTRODUCTION}

Electron emission from cold cathodes is currently an area of great interest especially because of its application to the flat panel display (FPD) industry. Many efforts have been applied to improve the emission from cold cathodes. ${ }^{1-12}$ These can be summarized into two categories: emission from conductive tips or edges (e.g., Refs. 1 and 2) and emission from flat conductive surfaces coated with films capable of reducing the electric field necessary for emission (e.g., Refs. 6,8 , and 10). Eventually both approaches were combined (e.g., Refs. 2, 10, and 11) so emission could be obtained at even lower electric fields (applied voltages).

Other constraints are also important to FPD, especially those related to vacuum performance. It has been shown ${ }^{2,13}$ that desorption from surfaces and poor vacuum conductance, both of which are normally present in conventional FPDs, can cause a substantial increase in pressure inside the FPD during its lifetime. The properties of low voltage phosphors ${ }^{13}$ and even the properties of the emitting surfaces themselves can vary significantly as a result of increased pressure. The pressure gradient normally associated with poor vacuum conductance can also introduce undesired brightness variations along the panel. ${ }^{2}$

We identified two possible gas pumping directions in a FPD: longitudinal and transverse. Longitudinal pumping is currently being used in conventional FPDs; that is, the pumping direction is parallel to the anode and cathode planes, since the getter is usually positioned on the edges of the FPD. This approach, considering a molecular pumping regime, leads to a vacuum conductance inversely propor-

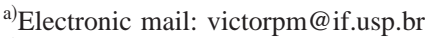

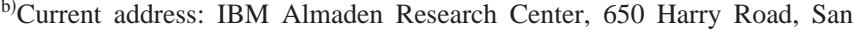
Jose, CA 95120.

${ }^{c}$ Electronic mail: mcsalvadori@if.usp.br
}

tional to the FPD width (for a fixed height) and directly proportional to the square of the anode-cathode spacing. On the other hand, in the transverse pumping, the pumping direction is normal to the anode and cathode planes, leading to a vacuum conductance that has a negligible dependence on the FPD width. And, if one considers only the vacuum conductance associated to the emission chamber (that is, the space between anode and cathode), the vacuum conductance would be inversely proportional to the anode-cathode spacing. But the implementation of the transverse pumping would introduce an important question: how can we pump gases through the cathode plane? Figure 1 shows a schematic representation of both pumping schemes.

A solution to achieve transverse pumping was previously proposed by some of the authors ${ }^{14}$ and consists of using diamond porous membranes as emitting cathodes. The field enhancement factor for the pores is at least comparable to that of other metallic edge, ${ }^{14}$ and the porosity itself promotes a substantial increase in vacuum conductance through the cathode plane. In such membranes, there is a direct correlation between the vacuum conductance and the area, because for a given pore density, the number of pores grows linearly with the FPD area. This fact will make it easier to scale up to larger area FPDs, and eliminate the need to increase the anode-cathode gap in order to produce high vacuum conductance. Maintaining a small interelectrode gap or decreasing it has the additional advantage of improved focusing. Last, but not less important in this concept is the fact that the porous membrane approach results in no long-range pressure gradients across the display area. In a previous performance test we proved that the porous diamond membrane is a promising emitter. Specifically, emission for a given pore density was shown to be superior to that of flat metallic surface, even when the former is coated with diamond-like carbon (DLC).

In the present work, we continue our investigation of dia- 

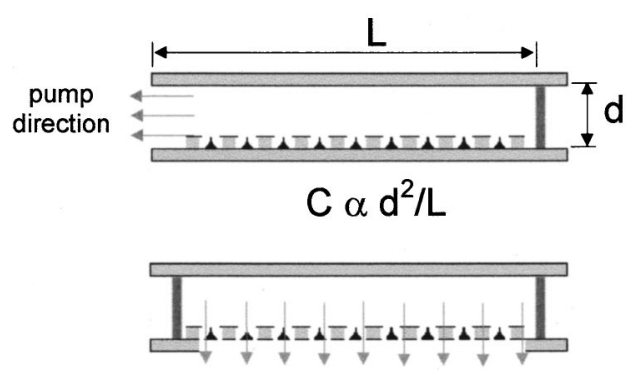

\section{$\mathrm{C} \alpha 1 / \mathrm{d}$ (disregarding the bottom surface)}

FIG. 1. Schematic of a FPD: longitudinal (a) and transverse pumping (b). C is the vacuum conductance, $\mathbf{L}$ the FPD width, and $\mathbf{d}$ the anode-cathode spacing.

mond membrane emitters by characterizing the pore profile and the extent of metal film coating along the length of the inner pore walls. We also present photoemission electron microscopy (PEEM) images from the region around a pore demonstrating that there is indeed strong electron emission from such regions. A more detailed study of the emission is presented, including current versus time and current versus field curves.

\section{EXPERIMENT}

Porous diamond membranes consist of freestanding membranes with pores distributed in a regular array. Their manufacturing process is fully described elsewhere. ${ }^{15-18}$ Here we provide only a brief description of this process. The silicon wafer is patterned and etched so that a regular array of plateaus is left on the surface. Diamond is then deposited on these substrates using microwave assisted plasma chemical vapor deposition. After deposition, the top part of the protrusions is removed using a mechanical grinding procedure. The process is completed with the wet etching of the silicon mold, leaving a silicon frame in the outer regions, in order to increase the overall device resistance.

A thin layer of metallic material was deposited on its backside to form the conductive cathode. We chose tungsten deposited by metal plasma immersion ion implantation and deposition (MePIIID), ${ }^{19}$ since tungsten deposited through this method was proven to adhere well to diamond. ${ }^{20}$ Another reason for using MePIIID is its ability to deposit on surfaces that are not necessarily in direct line of sight of the plasma source. This becomes particularly important here, since the entire inner wall of the pores must be coated. During the deposition, the filtered cathodic arc plasma source was operated in a pulsed mode with $5 \mathrm{~ms}$ arc duration. The substrate was pulse biased during the entire deposition with $-2000 \mathrm{~V}$ for the first 1000 arc pulses, and $-100 \mathrm{~V}$ for the last 4000 arc pulses. The sample bias was pulsed with a duration of $4 \mu \mathrm{s}$. Pulse biasing guarantees a strong film adhesion to the membrane back surface, since the initial high bias voltage increases the atomic mixing between the diamond substrate and tungsten film. After that initial adhesion enhancement procedure the sample bias was reduced to avoid further sputtering of the deposited material. The distance between filter and sample was $20 \mathrm{~cm}$ and the chamber pressure was $8 \times 10^{-6}$ Torr. An S-shaped particle filter ${ }^{21}$ was used in the deposition process.

To study the local field emission properties of the membrane, a photoemission electron microscope (PEEM) was used. PEEM usually employs light sources such as ultraviolet (UV) lamps or synchrotron radiation to excite electron emission from a sample. The emitted electrons are imaged by an electron optics column, and the image depends on the radiation energy and sample emission properties. A strong electric field is applied between the sample and the electron optics column to accelerate the electrons into the column. This leads to topographical contrast because the electric field, and therefore the electron trajectories, are distorted at surface topographical features. If the sample surface is a strong field emitter, the electric field between the sample and electron optics column (typically of order $10 \mathrm{kV} / \mathrm{mm}$ ) is sufficient to produce enough electrons for the PEEM to be operated without light source. In this mode, solely the field emitted electrons can be imaged. One can distinguish between photoemission and field emission by switching off the light source. Both excitation modes (light and field) were applied in this study to identify local field emitting centers. The instrument used for these investigations was the PEEM2 facility at the Advanced Light Source, ${ }^{22}$ located at the Lawrence Berkeley Laboratory. In this analysis we used an $\mathrm{X}$-ray radiation of $350 \mathrm{eV}$, with a flux of about $10^{12}$ photons per second in a $50 \mu \mathrm{m} \times 50 \mu \mathrm{m}$ spot.

\section{RESULTS AND DISCUSSION}

We fabricated a diamond porous membrane with the following geometric characteristics: pore size about $15 \mu \mathrm{m}$, center-to-center pore distance of $200 \mu \mathrm{m}$, membrane thickness of $10 \mu \mathrm{m}$, and circular pore profile. The freestanding diameter was about $10 \mathrm{~mm}$. The pore size can be easily obtained in a range between 1 and $10^{2} \mu \mathrm{m},{ }^{16}$ while the pore to pore distance is a free parameter, provided it is not smaller than about 3 times the diamond thickness. In order to get denser porosity one can produce thinner diamond membranes (e.g., Ref. 23).

A top-view scanning electron microscope image of the membrane is shown in Fig. 2. The pore is approximately circular, with a diameter of $17 \mu \mathrm{m}$. A cross section scanning electron microscopy (SEM) image of a pore is also shown in Fig. 3. The quasicircular profile of the inner pore walls is evident. A slightly columnar structure normal to the diamond film surface can also be observed, as registered before by Ref. 24. It is also clear that the back of the membrane (the diamond nucleation surface) is much smoother than the growth side, and it conforms to the original silicon surface. The top part of the inner pore walls is not exactly perpendicular to the membrane plane. This results in the electrostatic enhancement factor being smaller than that of a vertical metallic edge emitter. Changes on the porous membrane manufacturing process are presently being developed to improve the pore morphology. The main aspect that has to be 


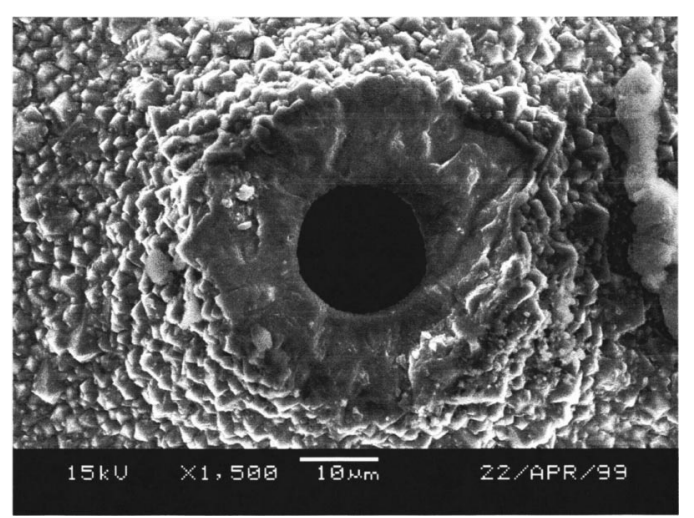

FIG. 2. SEM image showing the top view of a rounded pore in a diamond membrane.

changed is the process used to remove diamond from the top of the silicon plateaus. As discussed in Ref. 16, this process is responsible for pore size and shape irregularity. The alternatives to avoid this drawback that are currently under way are based on selective diamond nucleation and plasma etching.

As we have previously stated, the thin tungsten film is deposited on the smooth side of the diamond membrane, and it is important that this film extend all the way to the pore orifice. In order to verify the extent of surface coating, we have used electron dispersive $\mathrm{x}$-ray spectroscopy (EDX). The arrow in Fig. 3 shows the area corresponding to the EDX analysis. This technique identifies the elements present in the selected area, and based on these analyses, we concluded that tungsten was present on the top part of the inner walls. This fact identifies that the MePIIID technique was a suitable choice for tungsten deposition.

This cathode configuration is such that it is not obvious, $a$ priori, where the actual emission sites are. Disregarding the presence of diamond, one would expect that emission would occur primarily from the edges of the pores. The presence of diamond, however, may induce changes to the electrostatic field on the tungsten edges due to its dielectric constant. Moreover, the negative electron affinity of diamond can play

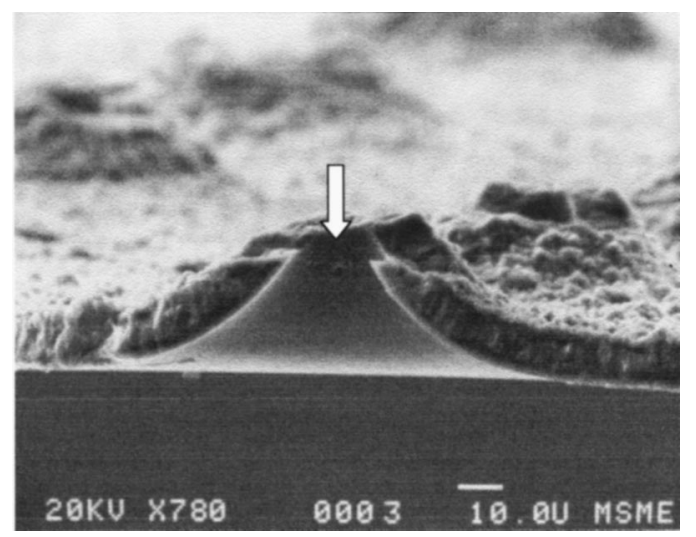

FIG. 3. SEM image showing the side view of a fractured membrane. The arrow shows the position where EDXS spectroscopy was done.

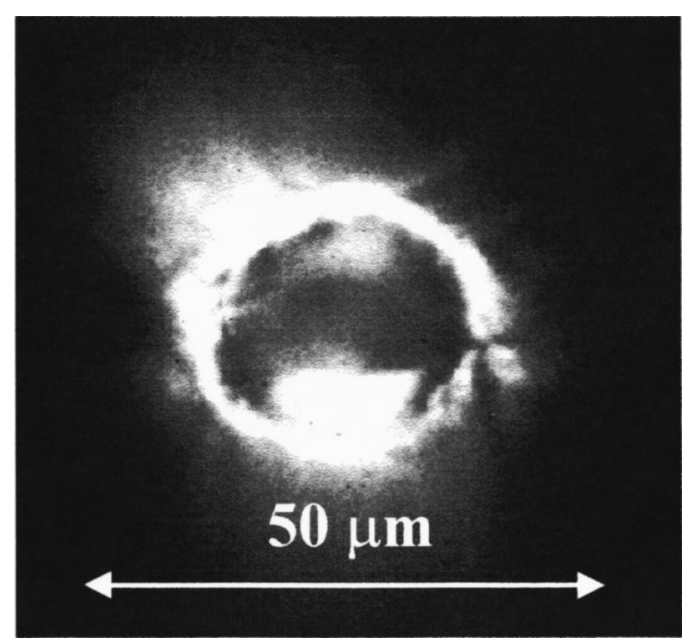

FIG. 4. PEEM image from a rounded pore (top view).

a very important role in reducing the field necessary for electron emission.

We performed PEEM analyses in order to locate the emission sites on the diamond structure. Images formed with photoemitted electrons provide an overall image of the structure, while images formed by field emitted electrons provide the image of the emission sites.

Figure 4 shows the photoemission image from a pore that presented no strongly enhanced electron field emission. The contrast in the image is due to topography. The electric field applied to the sample was about $10 \mathrm{kV} / \mathrm{mm}$. One can see clearly the rounded profile already observed in Fig. 2. Figure 5 shows another pore that exhibited strong field emission (onset field about $7.5 \mathrm{kV} / \mathrm{mm}$ ). The bright spot indicated in Fig. 5 remained bright when the light source was switched off, while the rest of the image disappeared. Comparing Figs. 4 and 5 one can speculate that the emission starts at different applied voltages for different pores. Field emission sites were not observed in the area between pores.

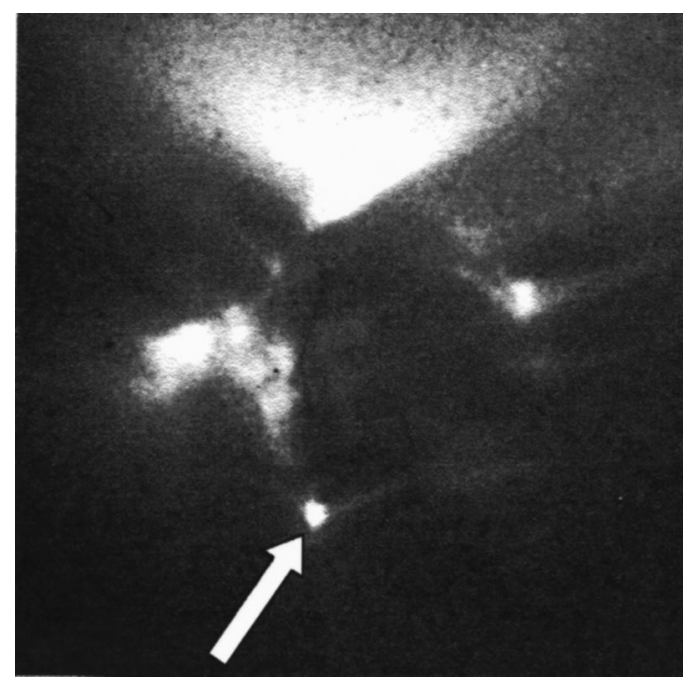

FIG. 5. Field excited image from a rounded pore similar to that shown in Fig. 4. The applied field was $7.5 \mathrm{kV} / \mathrm{mm}$. 


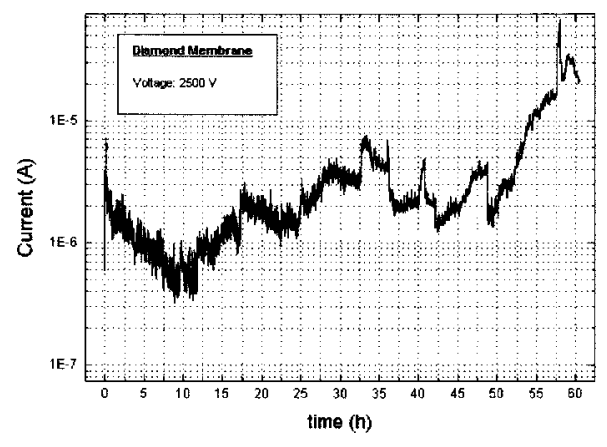

FIG. 6. Field emission stability measurement.

A very important aspect of the brightness of the field emission spot is its instability. This instability is compatible to the one usually observed in diamond emitters. Static images like Fig. 5 cannot show this aspect, but the dynamic image observed during the measurement shows that the field emission spots clearly have fluctuating brightness, while the contrast in images referent to photoemission is static. This fact coincides with the instability in current observed by applying a constant electric field to the membrane. Figure 6 shows the data related to these measurements. Figure 6 also shows an increase in emission current at a constant applied voltage after several hours of emission. The reason for this increase is still unknown and we speculate that the presence of diamond may account for it. Further investigation is required to explain this observation.

Figure 5 clearly shows that the emission does not take place uniformly around the pore for the field applied, but is concentrated in a small spot. This may be due to nonuniformity of the pore geometry as already observed by SEM in Fig. 2. Another explanation is the nonuniformity of the diamond film properties, which may cause preferential emission. The strong current density dependence on the electric field or work function causes the emission spots to appear at compositional, structural or morphological irregularities. This could also be responsible for the fact that emission was not obtained simultaneously from all pores at a given field.

However, if from one point of view, fields like $10 \mathrm{kV} / \mathrm{mm}$ did not produce uniform emission along the pore edge, the fact is that higher fields produced such bright emitting images that the contrast could not be adjusted to form a picture of the emitting sites. So we do not discard the possibility of occurrence of a more uniform emission along a pore edge for higher fields.

Figure 7 shows the current against electric field data obtained from one position at a membrane. A detailed description of the method used to plot Fig. 7 is given in Ref. 25. In short words we can say that the $I \times E$ curve is obtained from the $I \times V$ curve, considering an adjustable anode-cathode distance. After plotting an approach curve, a good estimation of this anode-cathode distance is obtained for each $I \times V$ curve measured. Subsequently this distance can be used to calculate the field, considering a parallel capacitor as the anodecathode geometry.

The main feature apparent in Fig. 7 is the spread of $I$

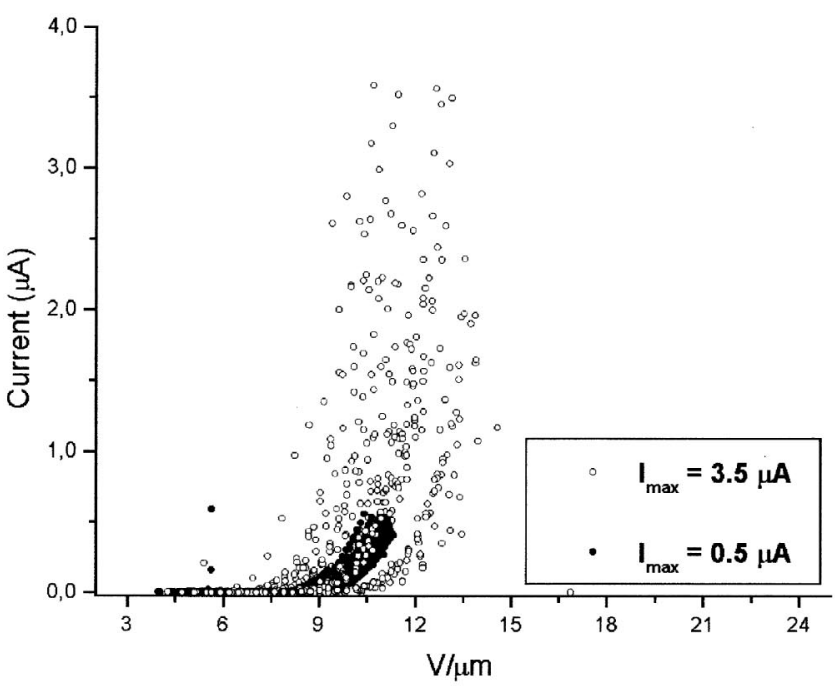

(a)

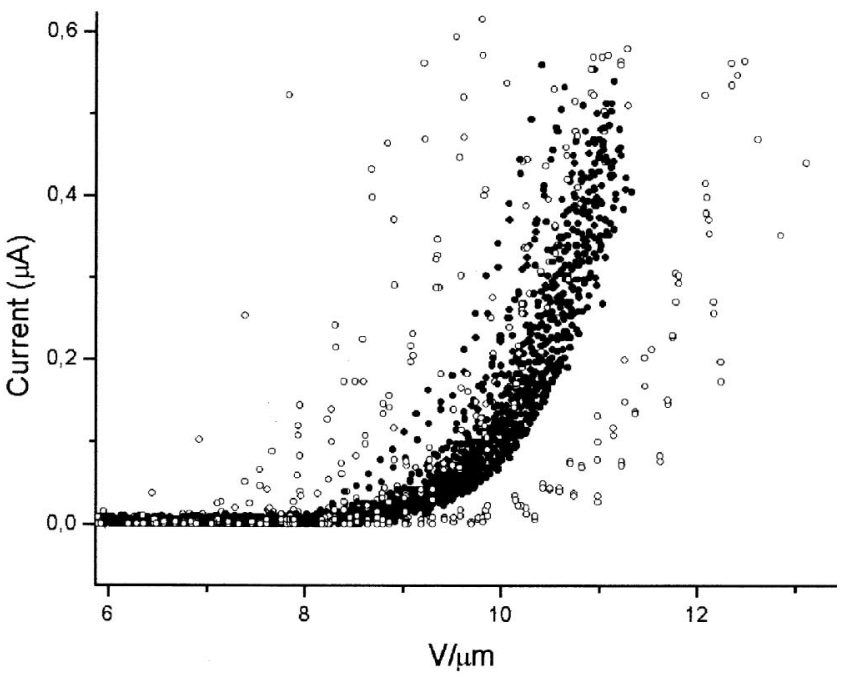

(b)

FIG. 7. Current $X$ electric field data $(I \times E$ curves). The opened circle data points refers to $I \times E$ curves for which current was limited below $3.5 \mu \mathrm{A}$, while closed circle refers to curve for which current was limited below 0.5 $\mu \mathrm{A}$. (a) Shows a general view and (b) zooms a region of the same graph.

$\times E$ results, which is consistent with the instability observed in Fig. 6 taking into account that the total duration for acquiring the data shown in Fig. 7 is about $2 \mathrm{~h}$. At this point, it is important to say that the set of data points shown in Fig. 7 is subdivided into smaller sets, each one referring to a particular sequence of measurements, since the $I \times E$ curves were obtained individually. The open circles correspond to $I \times E$ curves that were obtained by sweeping the voltage so that the current would vary from 0 to $3.5 \mu \mathrm{A}$. The closed circles were obtained with current ranging from 0 to $0.5 \mu \mathrm{A}$. For the geometry used, $3.5 \mu \mathrm{A}$ of total current represents, roughly, $10^{-1} \mu \mathrm{A}$ per pore, while $0.5 \mu \mathrm{A}$ of total current represents about $10^{-2} \mu \mathrm{A}$ per pore.

It is clear from Fig. 7(a) that the spread of open circles is bigger than the spread of closed ones. This fact is probably due to changes that high currents per pore can produce in the 
emitting characteristics. $I \times E$ curves obtained with lower currents per pore (closed circles) are more reproducible, and therefore result in less scattering of the data. Figure 7(b) is a zoom of a region of Fig. 7(a), and the density of closed circles can be observed in a much more clear way, showing that the statistics are very meaningful.

\section{CONCLUSIONS}

In this article we have successfully prepared emitters based on metallized porous diamond membranes. This approach has significant advantages regarding vacuum performance, and we have observed here that field emission occurs at fields below $10 \mathrm{kV} / \mathrm{mm}$. Early field and vacuum calculations $^{14}$ demonstrated the usefulness of this concept. We observed an important current instability, and also that the $I \times E$ curves are much more reproducible if low currents per pore are induced.

PEEM results showed that the region around the pore is indeed the place where the emitting sites are located, but there was not enough spatial resolution in the microscope to determine whether emission was from the edges of the metallic film or from the nearby diamond. No emission was observed between pores.

\section{ACKNOWLEDGMENTS}

This work was supported, in part, by FAPESP and CNPq (Brazil) and in part by the DOE (U.S.) Contract No. DEAC03-76SF00098. The authors would like to also thank Professor Francisco Tadeu Degasperi (FATEC/SP) and the institutions IM/CTI and LSI/EPUSP from Brazil.
${ }^{1}$ I. Brodie and C. A. Spindt, Adv. Electron. Electron Phys. 83, 1 (1992). ${ }^{2}$ D. Temple, Mater. Sci. Eng., R. 24, 185 (1999).

${ }^{3}$ B. R. Johnson, A. I. Akinwande, and D. Murphy, J. Vac. Sci. Technol. B 15, 535 (1997)

${ }^{4}$ J. G. Fleming, D. A. A. Ohlberg, T. Felter, and M. Malinowski, J. Vac. Sci. Technol. B 14, 1958 (1996).

${ }^{5}$ O. Gröning, O. M. Küttel, P. Gröning, and L. Schlapbach, Appl. Phys. Lett. 71, 2253 (1997).

${ }^{6}$ Z. Feng, I. G. Brown, and J. W. Ager III, J. Mater. Res. 10, 1585 (1995).

${ }^{7}$ F. Y. Chuang, H. F. Cheng, C. Y. Sun, and I. N. Lin, J. Vac. Sci. Technol. B 15, 2072 (1997).

${ }^{8}$ D. S. Mao et al., Diamond Relat. Mater. 8, 52 (1999).

${ }^{9}$ F. Y. Chuang, W. C. Wang, F. Cheng, C. Y. Sun, and I. N. Lin, J. Vac. Sci. Technol. B 15, 2072 (1997).

${ }^{10}$ V. V. Zhirnov and J. J. Hren, Mater. Res. Bull. 23, 42 (1998).

${ }^{11}$ V. V. Zhirnov, A. B. Voronin, E. I. Givargizov, and A. L. Meshcheryakova, J. Vac. Sci. Technol. B 14, 2034 (1996).

${ }^{12}$ T. K. Ku et al., Thin Solid Films 290/291, 176 (1996).

${ }^{13}$ P. H. Holloway, J. Sebastian, T. Trottier, S. Jones, H. Swart, and R. O. Petersen, Symposium H, Flat Panel Display Materials, Spring Meeting Symposium Proceedings (Materials Research Society, Pittsburgh, 1996).

${ }^{14}$ V. P. Mammana, F. T. Degasperi, O. R. Monteiro, J. H. Vuolo, M. C. Salvadori, and I. G. Brown, J. Vac. Sci. Technol. A 18, 1818 (2000).

${ }^{15}$ V. P. Mammana, R. D. Mansano, P. Verdonck, A. Pavani, and M. C. Salvadori, Diamond Relat. Mater. 6, 1824 (1997).

${ }^{16}$ V. P. Mammana, M. C. Salvadori, and I. G. Brown, Thin Solid Films 308/309, 281 (1997).

${ }^{17}$ M. C. Salvadori, M. Cattani, V. Mammana, O. R. Monteiro, J. W. Ager, and I. G. Brown, Thin Solid Films 290/291, 157 (1996).

${ }^{18}$ V. P. Mammana, S. Silva, R. D. Mansano, P. Verdonck, A. Pavani, M. C. Salvadori, and I. G. Brown, Thin Solid Films 353, 239 (1999).

${ }^{19}$ Ian Brown, Annu. Rev. Mater. Sci. 28, 243 (1998).

${ }^{20}$ O. R. Monteiro, M. C. Salvadori, M. Cattani, V. Mammana, and I. G. Brown, Thin Solid Films 308/309, 235 (1997).

${ }^{21}$ S. Anders, A. Anders, M. R. Dickinson, R. A. MacGill, and I. G. Brown, IEEE Trans. Plasma Sci. 25, 670 (1997).

${ }^{22}$ S. Anders et al., Rev. Sci. Instrum. 70, 3973 (1999).

${ }^{23} \mathrm{H}$. Noguchi et al., J. Vac. Sci. Technol. B 16, 2772 (1998).

${ }^{24}$ V. J. Trava-Airoldi, E. J. Corat, A. F. V. Pena, N. F. Leite, V. Baranauskas, and M. C. Salvadori, Diamond Relat. Mater. 4, 1255 (1995).

${ }^{25}$ S. Dimitrijevic, J. C. Withers, V. P. Mammana, O. R. Monteiro, J. W. Ager III, and I. G. Brown, Appl. Phys. Lett. 75, 2680 (1999). 